period may persist into adulthood, increasing chronic disease risk in later life. A better understanding of the underlying trajectories of sugar consumption across adolescence and early adulthood will help inform appropriate interventions for this age group. This systematic review investigated changes in intake of added sugars and sugary foods and drinks, and determinants of such changes, between the ages of 13 and 30 years.

Methods In accordance with the registered protocol (PROSPERO: CRD42015030126), 7 electronic databases were searched in January 2016 for longitudinal studies of diet during adolescence or early adulthood. The papers retrieved were screened for studies including multiple measures of intake of sugars or sugary foods or drinks in cohort participants between the ages of 13 and 30 years. Data from included studies were extracted and analysed using random-effects meta-analysis, by the three main nutrient and food group categories identified.

Results We identified 23 papers reporting longitudinal data on intake of added sugar or sucrose $(n=5)$, sugar-sweetened beverages $(\mathrm{SSBs})(\mathrm{n}=20)$ and/or confectionery $(n=9)$. Eight papers reported data from the US, with Norway, Sweden and Australia also contributing several papers. On average, we found a per-year of age decrease in added sugar or sucrose intake $(-0.28 \%$ total energy intake $(95 \%$ CI $-0.44 ;-0.12))$, a decrease in confectionery consumption $(-0.20$ servings/week $(95 \%$ CI $-0.41 ;-0.001)$ ) and a non-significant decrease in SSB consumption (-0.15 servings/week (95\% CI -0.32 ; 0.02)). A small number of studies reported associations between behavioural determinants (e.g. screen time and fast food consumption) and change in intake of added sugar. Only three papers reported data beyond the age of 21 which weakens applicability of these findings to the early adulthood years.

Conclusion This review demonstrates a decrease in added sugar intake from adolescence to early adulthood, which may suggest an opportunity to capitalise on such changing preferences with interventions to further improve dietary choices within this age range. Improved longitudinal data is needed to further develop our understanding of changes in added sugar consumption into early adulthood and determinants of these changes.

\section{OP47 RELATIONSHIPS BETWEEN SUGAR INTAKE FROM SWEET FOOD AND BEVERAGES, COMMON MENTAL DISORDER AND DEPRESSION: PROSPECTIVE FINDINGS FROM THE WHITEHALL II COHORT STUDY}

A Knüppel*, MJ Shipley, CH Llewellyn, EJ Brunner. Department of Epidemiology and Public Health, University College London, London, UK

\subsection{6/jech-2017-SSMAbstracts.47}

Background In the face of high rates of mood disorders and an overconsumption of sugar, intake of sweet food, beverages and added sugars has been linked with depressive symptoms in several populations. It remains unclear if this association is causal or due to reverse causation (the influence of mood on sugar intake). Our study aimed to investigate systematically cross-sectional and prospective associations between sugar intake from sweet food/beverages, common mental disorder (CMD) and depression, and to examine the role of reverse causation as potential explanation for the observed linkage.
Methods We analysed repeated measures (23 245 person-observations) from the Whitehall II cohort study. The study recruited non-industrial civil servants from 1985 to 1988 . The most recent data used in this analysis was collected in 2013. CMD was measured with the 30-item General Health Questionnaire and depression with the 20-item Centre for Epidemiologic Studies Depression scale. Sugar intake from sweet food/beverages was assessed using food frequency questionnaires. Prospective analyses included 2, 5 and 10 year followup periods. We modelled associations using random effects regression using Stata 14.

Results Cross-sectional analyses showed positive associations. In prospective analyses we found a positive association of sugar intake from sweet food/beverages with incident CMD in men and with recurrent depression in women. Men in the highest tertile of intake had a 24\% increased odds of incident CMD after 5 years (95\% CI: 1.02, 1.48) independent of health behaviours, socio-demographic and diet-related factors, adiposity and other diseases (fully adjusted model). Women had a $36 \%$ increased odds for recurrent depression per $30 \mathrm{~g}$ increment $(95 \% \mathrm{CI}: 1.03,1.80)$ in fully adjusted models, associations using tertiles of sugar intake from sweet food/beverages were similar but not statistically significant when adjusted for diet-related factors. Notably, neither CMD nor depression predicted changes in sugar intake from sweet food/beverages. Conclusion Our findings using repeated measures follow-up data over 22 years suggest an adverse effect of sugar intake from sweet food/beverages on long-term psychological health. These results add further support for public health interventions which promote reduced sugar intake to improve overall health.

\section{OP48 AGE, SEX AND SOCIOECONOMIC INEQUALITIES IN FRUIT AND VEGETABLE INTAKE IN UK ADULTS, 1986- 2012}

A Yau*, J Adams, P Monsivais. Centre for Diet and Activity Research, MRC Epidemiology Unit, University of Cambridge, Cambridge, UK

\subsection{6/jech-2017-SSMAbstracts.48}

Background Low fruit and vegetable (FV) consumption is associated with cardiovascular disease and some cancers. The UK recommendation is that adults should consume at least $400 \mathrm{~g}$ $(5 \times 80 \mathrm{~g}$ portions) of $\mathrm{FV}$ per day. However, the most recent available data indicate that over $80 \%$ of UK adults do not adhere to the '5-a-day' recommendation. This study investigates whether the level of adherence has changed over time. Further, we look at number of FV portions consumed by age, sex and socioeconomic position across the three time points between 1986 and 2012.

Methods We used three surveys for this repeated cross-sectional analysis: the National Diet and Nutrition Survey (2000/ 01 and 2008/12) and a similar survey from 1986/87, the Dietary and Nutritional Survey for British Adults. These surveys employ multistage random sampling. FV intake was assessed using food diaries and demographic information collected by an interviewer. We included adults aged 19 to 64, who adequately completed a food diary, which gave sample sizes of 2047, 1724 and 1512, in 1986/87, 2000/01 and 2008/12 respectively. To test for socioeconomic inequalities, respondents were categorised into four groups (professional/managerial (I/ II); skilled non-manual (IIINM), skilled manual (IIIM); partly 
skilled/unskilled (IV/V)) based on the Registrar General Social Class classification and we looked at absolute and relative differences between I/II and IV/V. To test differences in mean intake by demographic characteristics, we used t-tests and linear regression. All analyses were conducted in StataSE 13.

Results Overall FV intake increased by 20\% from 1986/87 (2.67 portions, 95\% confidence interval [2.59,2.74]) to 2008/ 12 (3.21 portions [3.10,3.32]), but still fell short of the recommended 5 portions. We found higher FV intake in socioeconomic group I/II compared to IV/V across all years (difference of $0.96-1.28$ portions; $26.09 \%-36.36 \%$ ). FV intake also differed by age across all surveys, with older respondents eating more than younger respondents (1.03-2.08 portions; 51.76\%-129.19\%). Men ate significantly more FV than women in $1986 / 87$ (0.18 portions; $6.52 \%)$ but there was no significant difference in the latter time points.

Conclusion While FV consumption has increased in the UK since 1986, and sex differences in consumption have disappeared, socioeconomic and age-related inequalities persist. Population-level strategies to improve diet and increase FV intake are still needed in the promotion of public health. In order to redress inequalities, it is crucial that these strategies are at least or more effective in lower versus higher socioeconomic groups, and in younger versus older adults.

\section{Ageing 1}

\section{OP49 ASSOCIATION BETWEEN TEMPERATURE AND DEATH AMONG ELDERLY PEOPLE IN ENGLAND 2012/13-2013/ 14: A CASE-CROSSOVER DESIGN}

${ }^{1} \mathrm{P}$ Tammes ${ }^{*},{ }^{2} \mathrm{C}$ Sartini, ${ }^{3}$ I Preston, ${ }^{1} \mathrm{AD}$ Hay, ${ }^{4} \mathrm{D}$ Lasserson, ${ }^{1} \mathrm{RW}$ Morris. ${ }^{1}$ School of Social and Community Medicine, University of Bristol, Bristol, UK; ${ }^{2}$ Department of Primary Care and Population Health, University College London, London, UK: ${ }^{3}$ Household Energy Services, Centre for Sustainable Energy, Bristol, UK; ${ }^{4}$ Nuffield Department of Medicine, University of oxford, Oxford, UK

\subsection{6/jech-2017-SSMAbstracts.49}

Background Around 24000 extra deaths occur annually in winter in England and Wales. NICE guidance suggests GPs should identify patients most at risk. We investigated whether socio-demographic and clinical characteristics could predict cold-related mortality.

Methods Data on over 500000 patients aged $65+$ from the Clinical Practice Research Datalink (CPRD) were linked with ONS death registration, yielding 34777 patients who died between April 2012 and March 2014. We used daily temperature data from the Met Office to calculate (i) absolute mean temperature and (ii) difference from average monthly temperature (relative temperature) for the date of death and three days previously. In a case-crossover analysis, we also calculated both temperature measures for the 14th day before and the 14th day after the date of death. Patients assumed to live in an institution were identified using the CPRD family number. From linked Hospital Episode Statistics, we determined whether an emergency hospital admission occurred two years before death to indicate previous health status. Deprivation level and house energy efficiency were determined from patient's and practice's Lower Super Output Area respectively: the latter used information from the Centre for Sustainable Energy. Conditional logistic regression models were applied to estimate the odds ratio (OR) of death associated with temperature and interactions between temperature and sociodemographic, medical and house quality characteristics were expressed as relative odds ratios (RORs).

Results Higher absolute temperature was associated with lower risk of death (OR 0.985 per $1^{\circ} \mathrm{C}$; 95\% CI 0.975-0.992; $\mathrm{p}=<0.001)$. There was weak evidence of a positive association between risk of death and higher relative temperature (OR 1.008 per $1^{\circ} \mathrm{C}$; 95\% CI $\left.0.999-1.017 ; \mathrm{p}=0.056\right)$. No interactions were found between temperature measures and age, gender, living in urban/rural areas, deprivation level, or house energy efficiency in either bivariable or multivariable analyses. There was some evidence for a stronger effect of higher relative temperature for those living in an institution (ROR $1.025 ; 95 \%$ CI 1.002-1.048; $\mathrm{p}=0.03$ ), but not in multivariable analysis. Effects of temperature measures differed between those who had none vs at least one previous emergency admission: ORs for absolute temperature were 0.970 and 0.988 per $1^{\circ} \mathrm{C}$, with ROR 1.018, 95\% CI 0.998-1.039, $\mathrm{p}=0.079$. For relative temperature ORs were 1.033 and 1.003, with ROR 0.974, 95\% CI 0.951, 0.997, p=0.025, suggesting less impact of relative temperature for those with a previous emergency admission.

Conclusion Recommendations for GPs to identify those at highest risk during cold weather cannot be supported by these results.

\section{OP50 INEQUALITIES IN TIME BETWEEN STOPPING WORK AND DEATH: ONS LONGITUDINAL STUDY}

ET Murray*, E Carr, P Zaninotto, J Head, N Shelton. Epidemiology and Public Health, University College London, London, UK

\subsection{6/jech-2017-SSMAbstracts.50}

Background Due to the financial challenges of increasing life expectancy, many industrialised countries are raising state pension eligibility ages (SPA). However, use of average life expectancy to calculate SPA ignores inequalities in health and life expectancy across socio-economic groups.

Methods Data was used from 120552 members of The Office for National Statistics Longitudinal Study (LS) who were aged 50-75 at the 2001 census and had information on work status at the 2011 census, or died between 2001 and 2011. First, multinomial logistic regression was used to examine the odds of being dead or not being in work at the 2011 census date, compared to being in paid work, by Registrar General occupational social classes. Then, right-censored linear regression was used to examine mean social class differences in age of stopping work, age of death, and years of life between stopping work and death before aged 85; separately for each outcome. All models were adjusted for gender and self-rated health status in 2001.

Results By the 2011 census date, $12.8 \%$ of the sample had died, 66.8\% were alive but not working and 20.4\% remained in work. Women were less likely to both to die in the next 10 years or to remain in work than men. Gender-adjusted analyses showed that lower social class was associated with earlier mean age of stopping work [unskilled manual vs professional: -2.76 years $(95 \% \mathrm{CI}-3.04,-2.48)]$ and mean age of dying (before aged 85) [-3.92 (-4.56,-3.27)]. Before aged 65 , work exit was mostly due to exit from paid employment, rather than mortality; although unskilled workers in this age group were still 1.92 times $(1.69,2.19)$ more likely to die 